Marc Großklos | Margrit Schaede

\title{
Gebäude mit
}

Energiegewinn

Schritte zum Energieüberschuss in Neubau und Bestand
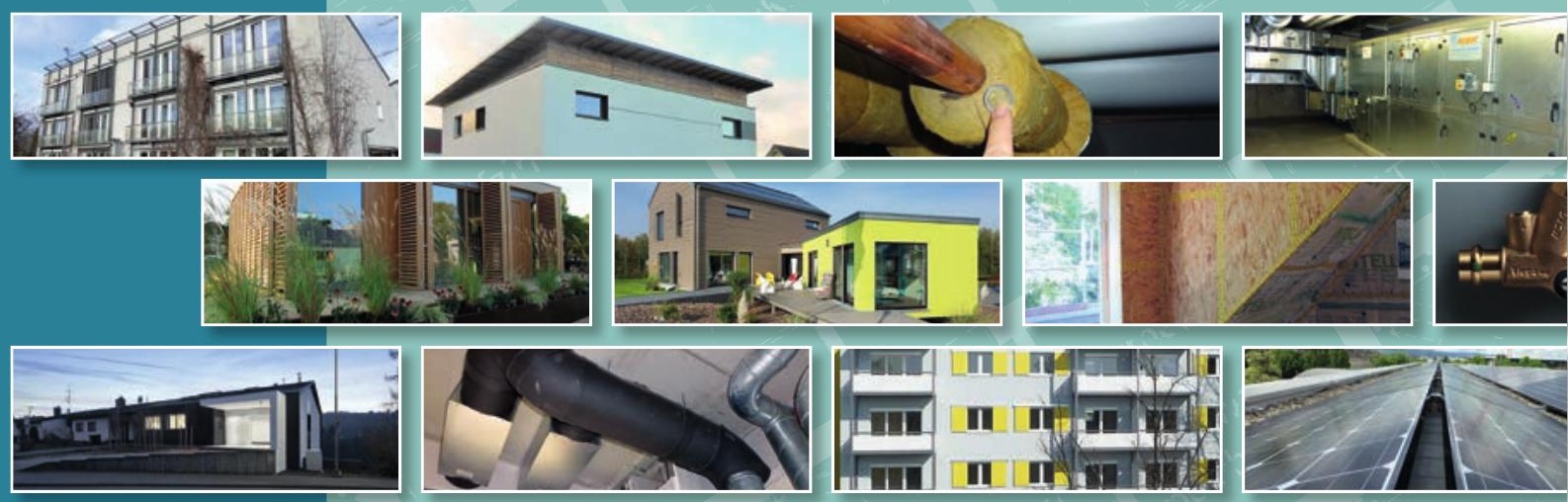

Fraunhofer IRB Verlag 
Marc Großklos, Margrit Schaede

Gebäude mit Energiegewinn 
Marc Großklos, Margrit Schaede

\section{Gebäude mit Energiegewinn}

Schritte zum Energieüberschuss in

Neubau und Bestand

Fraunhofer IRB Verlag 
Bibliografische Information der Deutschen Nationalbibliothek:

Die Deutsche Nationalbibliothek verzeichnet diese Publikation in der

Deutschen Nationalbibliografie; detaillierte bibliografische Daten sind

im Internet über www.dnb.de abrufbar.

ISBN (Print): 978-3-8167-9663-3

ISBN (E-Book): 978-3-8167-9664-0

Umschlaggestaltung: Martin Kjer

Herstellung: Andreas Preising

Satz: Reemers Publishing Services GmbH

Druck: Firmengruppe APPL, aprinta druck $\mathrm{GmbH}$, Wemding

Alle Rechte vorbehalten.

Die hier zitierten Normen sind mit Erlaubnis des DIN Deutsches Institut für Normung e. V. wiedergegeben. Maßgebend für das Anwenden einer Norm ist deren Fassung mit dem neuesten Ausgabedatum, die bei der Beuth Verlag GmbH, Burggrafenstraße 6, 10787 Berlin, erhältlich ist.

Dieses Werk ist einschließlich aller seiner Teile urheberrechtlich geschützt. Jede Verwertung, die über die engen Grenzen des Urheberrechtsgesetzes hinausgeht, ist ohne schriftliche Zustimmung des Fraunhofer IRB Verlages unzulässig und strafbar. Dies gilt insbesondere für Vervielfältigungen, Übersetzungen, Mikroverfilmungen sowie die Speicherung in elektronischen Systemen.

Die Wiedergabe von Warenbezeichnungen und Handelsnamen in diesem Buch berechtigt nicht zu der Annahme, dass solche Bezeichnungen im Sinne der Warenzeichen- und MarkenschutzGesetzgebung als frei zu betrachten wären und deshalb von jedermann benutzt werden dürften. Sollte in diesem Werk direkt oder indirekt auf Gesetze, Vorschriften oder Richtlinien (z. B. DIN, VDI, VDE) Bezug genommen oder aus ihnen zitiert werden, kann der Verlag keine Gewähr für Richtigkeit, Vollständigkeit oder Aktualität übernehmen. Es empfiehlt sich, gegebenenfalls für die eigenen Arbeiten die vollständigen Vorschriften oder Richtlinien in der jeweils gültigen Fassung hinzuzuziehen.

Die Inhalte stellen die persönliche Auffassung der Autoren dar.

(c) Fraunhofer IRB Verlag, 2016

Fraunhofer-Informationszentrum Raum und Bau IRB

Nobelstraße 12, 70569 Stuttgart

Telefon +49 $711970-2500$

Telefax $+49711970-2508$

irb@irb.fraunhofer.de

www.baufachinformation.de 


\section{Inhaltsverzeichnis}

1 Gebäude mit Energiegewinn - eine umsetzbare Vision? . . . . . . 11

1.1 Der Weg zum Ziel. . . . . . . . . . . . . . . . 15

1.2 Energieüberschuss als Anreiz. . . . . . . . . . . . . . 22

1.3 Offene Fragen im Zusammenhang mit Gebäuden mit Energiegewinn. . . . 23

1.4 Der Blick über das Gebäude hinaus. . . . . . . . . . . . . . 27

1.5 Von der Kohleheizung zum Energieüberschuss . . . . . . . . . 29

2 Bilanzierung von Gebäuden mit Energiegewinn. . . . . . . . . . 37

2.1 Bewertungsgrößen und Bilanzgrenzen . . . . . . . . . . 37

2.2 Weitere Aspekte der Bewertung von Gebäuden mit Energiegewinn. . . . 42

2.3 Bilanzierungsverfahren...................... 49

2.4 Bewertungsfaktoren für Energieträger . . . . . . . . . . . 56

2.5 Interaktion des Gebäudes mit dem elektrischen Netz. . . . . . . . . . . . 62

2.5.1 Auswirkungen elektrischer Energieerzeugung . . . . . . . . . . . . . . . . . . . . . . . 64

2.5.2 Auswirkungen elektrischer Verbraucher . . . . . . . . . . . . . . . 69

2.5.3 Bewertungsansätze für den Energieaustausch mit dem Stromnetz . . . . . 72

2.6 Definitionen und Standards für Gebäude mit Energiegewinn . . . . . . . . 73

2.6.1 »Niedrigstenergiegebäude« (Nearly Zero Energy Building - NZEB) . . . 73

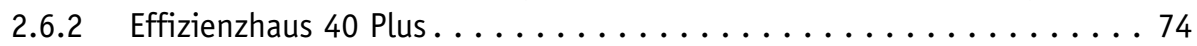

2.6 .3 Netto-Nullenergiehaus . . . . . . . . . . . . . . . . 74

2.6.4 Klimaneutrales Gebäude . . . . . . . . . . . . . . . . . . . 75

2.6.5 Effizienzhaus Plus: Definition der dena.............. 76

2.6 .6 Aktivhaus. ........................ 77

2.6.7 Effizienzhaus Plus: Definition des Bundesministeriums für Umwelt, Naturschutz, Bau und Reaktorsicherheit (BMUB)/Zukunft Bau . . . . . . 78

2.6.8 Passivhaus mit Energiegewinn . . . . . . . . . . . . . . 79

2.6.9 Passivhaus Plus und Passivhaus Premium . . . . . . . . . . . 79

2.6 .10 Sonnenhaus ........................ 80

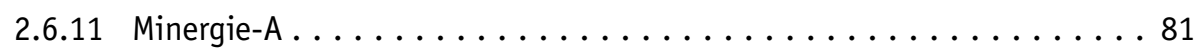

2.6 .12 Energieautarkes Haus. . . . . . . . . . . . . . . . 82

2.7 Empfehlungen für die Planung von Gebäuden mit Energieüberschuss . . . 82

3 Erster Schritt zum Energieüberschuss - die Reduktion des

Energiebedarfs. . . . . . . . . . . . . . . . . . . 89

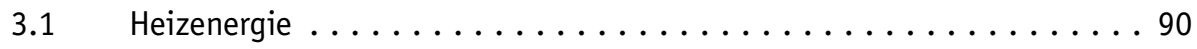

3.1 .1 Hochwirksamer Wärmeschutz . . . . . . . . . . . . . . 90

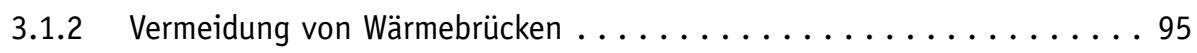

3.1 .3 Kompakte Bauweise. . . . . . . . . . . . . . . . 97

3.1 .4 Luftdichte Gebäudehülle. . . . . . . . . . . . . . . . 98 
3.1.5 Kontrollierte, bedarfsgerechte Wohnungslüftung. . . . . . . . . . . . 101

3.1.6 Ausnutzung passiv-solarer Gewinne . . . . . . . . . . . . . . . 103

3.1.7 Hocheffiziente, regenerative Heizung für den Restenergiebedarf . . . . 106

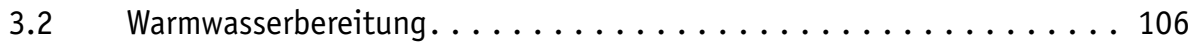

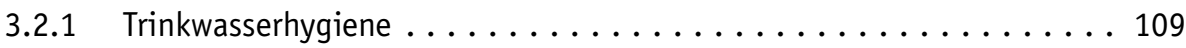

3.2.2 Anlagenarten für die Warmwasserbereitung . . . . . . . . . . . . . . . . . . . . . . 112

3.2.3 Effizienzmaßnahmen bei der Warmwasserbereitung . . . . . . . . . . 117

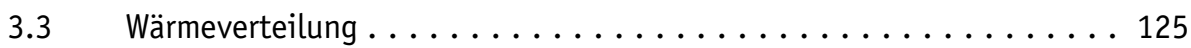

3.4 Haushaltsstrom . . . . . . . . . . . . . . . . . . . 130

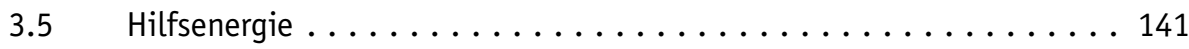

$3.6 \quad$ Nutzerverhalten . . . . . . . . . . . . . . . . . 146

4 Zweiter Schritt zum Energieüberschuss - Einsatz regenerativer

Energien . . . . . . . . . . . . . . . . . . 153

4.1 Wärmeerzeugung mit regenerativen Energiequellen . . . . . . . . . . 157

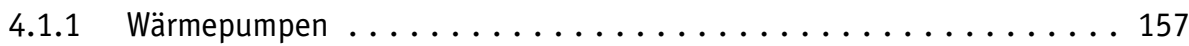

4.1 .2 Solarthermie........................... 179

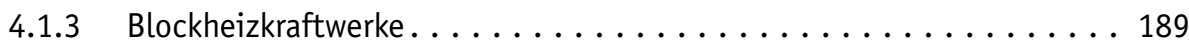

4.1 .4 Biomassekessel und - öfen. . . . . . . . . . . . . . . . 203

4.1.5 Gegenüberstellung der Techniken der Wärmeerzeugung . . . . . . . . . . 208

4.2 Thermische Energiespeicher. . . . . . . . . . . . . . . . 210

4.3 Stromerzeugung mit regenerativen Energien . . . . . . . . . . 217

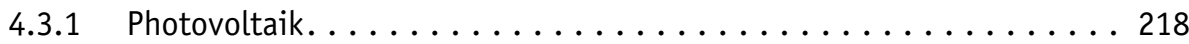

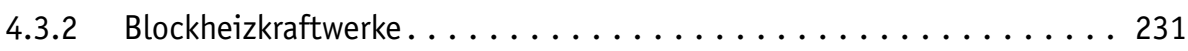

$4.3 .3 \quad$ Klein-Windkraft.......................... 235

4.3.4 Gegenüberstellung der Techniken der Stromerzeugung. . . . . . . . . . . . 241

$4.4 \quad$ Elektrische Energiespeicher . . . . . . . . . . . . . . . . . . 242

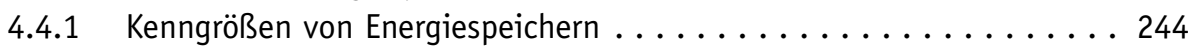

4.4.2 Arten elektrischer Energiespeicher . . . . . . . . . . . . . . . 245

4.4.3 Stationäre elektrische Energiespeicher für Gebäude. . . . . . . . . . . . 252

4.4.4 Auslegung von elektrischen Energiespeichern. . . . . . . . . . . . 258

$4.5 \quad$ Optimierter Anlagenbetrieb . . . . . . . . . . . . . . . . . 264

4.5.1 Erstellung eines Gesamtkonzeptes für die Anlagentechnik in der Planungsphase ........................ . . 265

4.5 .2 Inbetriebnahme ... . . . . . . . . . . . . . . 270

4.5.3 Anlagenüberwachung im Gebäudebetrieb. . . . . . . . . . . . . 271

5 Einflussgrößen auf den Energiegewinn von Gebäuden . . . . . . . . 273

5.1 Vorstellung der Modellgebäude Neubau und Bestand . . . . . . . . . 276

5.2 Berechnungsverfahren ..................... 282

5.3 Auswirkungen unterschiedlicher Randbedingungen auf die Energiebilanz . . . . . . . . . . . . . . . . . . . . . 284 
5.3.1 Einfluss des energetischen Standards des Gebäudes . . . . . . . . . . . 285

5.3.2 Einfluss der Gebäudegröße - bis zu welcher Größe ist ein Energieüberschuss möglich? . . . . . . . . . . . . . . . . . . . 292

5.3.3 Dachformen - Auswirkungen auf die Photovoltaikerzeugung. . . . . . . 295

5.3 .4 Photovoltaik-Fassaden . . . . . . . . . . . . . . . . . 302

5.3 .5 Verschattung des Gebäudes..................... 303

5.3.6 Vergleich unterschiedlicher Anlagenkonzepte ............ 306

5.3 .7 Gebäudeausrichtung. . . . . . . . . . . . . . . . . . 311

5.3 .8 Standort und klimatische Bedingungen . . . . . . . . . . . . . 314

5.3 .9 Einfluss veränderten Nutzerverhaltens . . . . . . . . . . . 317

6 Beispiele von Gebäuden mit Energiegewinn . . . . . . . . . . . . . 321

6.1 Neubau Einfamilienhaus mit Wärmepumpe. . . . . . . . . . . . . . . . 321

6.2 Neubau Einfamilienhaus mit Solarthermie . . . . . . . . . . . . . . 324

$6.3 \quad$ Neubau Zweifamilienhaus . . . . . . . . . . . . . . . . . . . . . . . . . . . . 327

6.4 Sanierung Einfamilienhaus . . . . . . . . . . . . 330

$6.5 \quad$ Neubau Mehrfamilienhaus. . . . . . . . . . . . . . . . . . 333

6.6 Sanierung Mehrfamilienhäuser. . . . . . . . . . . . . . 337

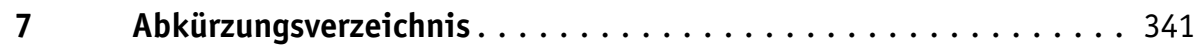

$8 \quad$ Literaturverzeichnis . . . . . . . . . . . . . . 347

Stichwortverzeichnis ... . . . . . . . . . . . . 367 


\section{Vorwort}

Die Wörter »Gewinn« und »Überschuss« beschreiben einen Ertrag nach Abzug des Aufwandes. Synonym kann auch das Wort »Plus« verwendet werden. Diese Bezeichnungen aus der Finanzwelt werden seit einigen Jahren auch bei besonders innovativen Gebäuden eingesetzt, die in ihrer Energiebilanz einen Ertrag liefern, also mehr Energie produzieren, als sie verbrauchen. Dies bedeutet jedoch nicht, dass sie zu jeder Zeit einen Überschuss erzielen, also autark sind. Dennoch bleibt am Ende ein Überschuss, der sich auch finanziell - zum Beispiel als Überweisung vom Stromversorger - in der eigenen Geldbörse positiv auswirken kann.

Eine andere Bedeutung des Wortes Gewinn beschreibt aber auch den gesellschaftlichen Nutzen oder einen ideellen Mehrwert. Auch diese Analogie passt gut zu den Gebäuden, die in diesem Buch beschrieben werden, da sie über die individuellen Vorteile hinaus einen Beitrag zu gesellschaftlichen Zielen leisten können und sollen. Dazu gehört unter anderem die in Deutschland seit dem Jahr 2011 angestoßene Energiewende, die allzu oft nur auf den Bereich der elektrischen Energie und deren Erzeugung reduziert wird. Damit die Energiewende gelingt, müssen jedoch Energieeffizienz und regenerative Energieversorgung gemeinsam umgesetzt werden, ergänzt um die Frage, welcher Komfort tatsächlich benötigt wird. Sowohl für die Energiewende als auch zur Erreichung der langfristigen Klimaschutzziele, um den weltweiten Anstieg der Treibhausgas-Emission zu begrenzen, können Gebäude mit Energiegewinn mittelfristig einen Beitrag leisten.

Gebäude mit Energiegewinn stellen zwar nicht die einzige Lösung zur Bewältigung der Herausforderungen dar, sie können aber einen Anstoß für die wichtige und erforderliche Weiterentwicklung bei Neubauten und vor allem bei der Sanierung liefern. Denn der größte Teil des Gebäudebestandes, den wir im Jahr 2050 bewohnen werden, ist bereits heute gebaut. Allerdings ist er bei Weitem noch nicht in dem energetischen Zustand, der langfristig zur Erreichung der Energiewende und der Klimaschutzziele erforderlich ist. Neue Konzepte, wie die Gebäude mit Energiegewinn, sind hier ein wichtiger Baustein. Schließlich sind Weiterentwicklungen im Baubereich auch für die Industrie von Interesse. Auch in anderen Ländern werden Konzepte für Gebäude mit Energiegewinn interessiert verfolgt und umgesetzt und können Exportchancen für die Wirtschaft erhöhen.

In den vergangenen Jahren wurden sehr unterschiedliche Begriffe für Gebäude mit Energiegewinn geprägt - z. B. Plusenergiehaus, Effizienzhaus Plus, Aktivhaus - die alle eine ähnliche Zielrichtung aufweisen, jedoch unterschiedlich definiert sind. Die Ansätze unterscheiden sich in ihrer Bilanzgrenze, im Bilanzumfang sowie den eingesetzten Techniken. Das vorliegende Buch will die unterschiedlichen Ideen und Konzepte von Gebäuden mit Energiegewinn vorstellen und Randbedingungen und 
Lösungen nicht nur für Neubauten, sondern auch für die Sanierung bestehender Häuser aufzeigen. Die vorgestellten Techniken sind alle am Markt verfügbar, die Konzepte können bereits heute umgesetzt werden. Das Buch konzentriert sich hierbei auf Wohngebäude und behandelt unterschiedliche Gebäudegrößen vom Einfamilienhaus bis hin zum großen Mehrfamilienhaus. Auch bei Nichtwohngebäuden wie Bürogebäuden, Fabriken oder Schulen sind Konzepte zur Erreichung eines Energieüberschusses möglich und auch schon umgesetzt worden. Diese würden jedoch den Rahmen dieses Buches sprengen und werden nicht im Detail erläutert.

Die Idee zu diesem Buch entstand bei Ausarbeitungen der Autoren zu innovativen Gebäuden mit Energiegewinn sowie aus Erfahrungen von konkreten Modernisierungsund Neubauvorhaben, die sie in den letzten Jahren wissenschaftlich begleitet haben. Hier sind besonders in Frankfurt das Passivhaus mit Energiegewinn Cordierstraße 4 sowie die Sanierung von sieben Mehrfamilienhäusern in der Rotlintstraße 116-128 als $\mathrm{CO}_{2}$-neutrale-Gebäude zu nennen. Beide Vorhaben wurden vom Hessischen Ministerium für Wirtschaft, Energie, Verkehr und Landesentwicklung unterstützt. Die Autoren möchten sich hierfür beim fördernden Ministerium bedanken. Weiterer Dank gilt Britta Stein und Dr. Falk Auer für die Durchsicht einzelner Kapitel des Manuskripts sowie allen Kollegen im Institut Wohnen und Umwelt für die konstruktive Kritik bei der Erarbeitung dieses Buches und die Bereitschaft, die eigenen Ergebnisse zur Verfügung zu stellen. Ein besonderer Dank gilt Rolf Born, der viele der verwendeten Grafiken erstellt hat.

Das Buch gliedert sich in sechs Hauptkapitel. Zu Beginn werden die Gründe diskutiert, warum und wo Gebäude mit Energiegewinn vorteilhaft sind und aus welcher Motivation sie gebaut werden. Kapitel 2 geht auf die Bilanzierung von Gebäuden mit Energiegewinn ein, stellt unterschiedliche Definitionen vor und leitet Empfehlungen für die Planung und Umsetzung ab. Zuerst sollte der Energiebedarf so weit wie möglich reduziert werden, was in Kapitel 3 beschrieben wird. Anschließend wird in Kapitel 4 dargestellt, wie die Deckung des verbleibenden Energiebedarfs und die Erzeugung eines Überschusses mit der Anlagentechnik zur Nutzung regenerativer Energien erfolgt. Welche Randbedingungen die Energiebilanz eines Gebäudes mit Energiegewinn in welcher Weise beeinflussen und wie ein Überschuss bei unterschiedlichen Gebäudegrößen erreicht werden kann, wird in Kapitel 5 beschrieben. Schließlich folgt in Kapitel 6 die Vorstellung mehrerer realisierter Beispielgebäude, die auch die Bandbreite der umgesetzten Konzepte und die architektonischen Möglichkeiten aufzeigen. Auch in den Kapiteln zuvor wird immer wieder auf diese Beispiele eingegangen.

Wir wünschen Ihnen nun eine spannende Lektüre und viele Anregungen zur Einarbeitung in die Materie, für eigene Bauvorhaben, für die Weiterentwicklung der vorgestellten Ansätze sowie als Diskussionsgrundlage zur Verbreitung nachhaltiger Konzepte.

Darmstadt, Mai 2016 